\title{
COMMENTARY
}

\section{Integrative Medicine in Clinical Practice: From Pattern Differentiation in Traditional Chinese Medicine to Disease Treatment}

\author{
LU Ai-ping (吕爱平) ${ }^{1}$ and CHEN Ke-ji (陈可冀) ${ }^{2}$
}

Pattern (syndrome) differentiation is the key theory in traditional Chinese medicine (TCM) and the important diagnostic principle for TCM therapy. More and more medical researchers recognize that the combination of disease diagnosis in biomedicine and pattern differentiation in TCM is essential for the clinical practice, and it has been a common practice model in China since it will produce better clinical effects ${ }^{(1)}$.

Pattern differentiation is mainly based on symptoms (including self-mentioned signs), tongue appearance and pulse palpation. The symptoms, tongue appearance and pulse palpation are often diversified. Some of them, not closely related to the disease diagnostic parameters, are less focused upon by modern medicine, such as thirst and turbid urine and yellow tongue fur in rheumatoid arthritis $(\mathrm{RA})^{(2)}$.

Although symptoms are diversified in a disease, they can be clustered into specific groups with biostatistical approaches, even though traditionally they are classified into groups based on TCM theory and clinical experiences. A multicenter RCT study on RA had shown that 18 symptoms (5 related to joint, 13 not related to joint but focused by TCM) in 396 patients could be classified into 4 factors (symptom combinations) with factor analysis. The symptom combinations are similar to the patterns differentiated by TCM theory in RA patients, which divides RA into three basic patterns: cold, hot and deficient patterns ${ }^{(3)}$.

TCM patterns in a disease, such as RA, should have bases in the view of biomedical sciences. The gene profile differences between the cold and hot patterns of RA patients might mainly refer to the amino acid and fat metabolism ${ }^{(4)}$. Another research on chronic gastritis showed that the TCM symptom groups in chronic gastritis were positively correlated with gastric mucosal immune reactions ${ }^{(5)}$.

Pattern differentiation, as an idea about classification, might be helpful in making innovations in biomedical sciences. Based on the TCM pattern differentiation, the severe cartilage erosion in the later stages of RA patients was regarded as the blood stasis and deficiency pattern in TCM. It was proven that blood stasis and deficiency pattern in TCM was related to blood rheology and immune regulation ${ }^{(6)}$. Thus, it is reasonable to make the hypothesis about positive relationships among $\lg \mathrm{A}$, platelet and cartilage erosion. The changes in peripheral IgA level and platelet number were positively correlated with the grade of cartilage damage in active RA patients which supported the hypothesis ${ }^{(7,8)}$.

More importantly, pattern differentiation will help improve the clinical efficacy in clinical practice since it further specifies the indication with TCM classification. A clinical research shows that the better effective treatment rate could be achieved from the RA patients with some specific symptoms and the results suggest that RA patients should be treated differently based on their patterns differentiated ${ }^{(3)}$.

Above all, pattern differentiation based on TCM information such as symptoms is important for further stratification of disease, which will help improve the clinical practice in the treatment of disease with further specified indications of the therapy.

\section{REFERENCES}

1. Lu AP, Ding XR, Chen KJ. Current situation and progress in integrative medicine in China. Chin J Integr Med 2008;14:234-240.

2. Lu AP, Jia HW, Xiao C. Theory of traditional Chinese medicine and therapeutic method of diseases. World $\mathrm{J}$ Gastroenterol 2004;10:1854-1856.

3. He YT, Lu AP, Zha QL, Yan XP, Song YJ, Zeng SP, et al. Correlations between symptoms as assessed in traditional chinese medicine (TCM) and ACR20 efficacy response: a comparison study in 396 patients with rheumatoid arthritis treated with TCM or Western medicine. J Clin Rheumatol 2007;13:317-321.

4. Lu C, Xiao C, Zhao LH, Zha QL, Yan XP, Wang JM, et al. Comparison of gene profile of peripheral $\mathrm{CD} 4^{+}$Iymphocytes in rheumatoid arthritis with cold and heat syndrome. Chin J Basic Med Tradit Chin Med (Chin) 2006;12:130-133.

5. Lu AP, Zhang SS, Zha QL, Ju DH, Wu H, Jia HW, et al. Correlation between CD4, CD8 cell infiltration in gastric mucosa, helicobacter pylori infection and symptoms in patients with chronic gastritis. World J Gastroenterol 2005;11:2486-2490.

6. Wu J, Chen KJ, Wu YJ. Phagocytosis of platelet in coronary heart disease patients with blood-stasis syndrome. Chin Med J (Engl) 1993;106:546-549.

7. He YT, Zha QL, Liu DY, Lu AP. Relations between serum IgA level and cartilage erosion in 436 cases of rheumatoid arthritis. Immunol Invest 2007;36:285-291.

8. Zha QL, He YT, Lu Y, Lu AP. Relationship between platelet counts and cartilage erosion in 436 cases of rheumatoid arthritis. Clin Chem Acta 2006;371:194-195.

(Received December 15, 2008) Edited by WANG Wei-xia

1. Chinese Association of Integrative Medicine, Beijing (100700), China; 2. Xiyuan Hospital, China Academy of Chinese Medical Sciences, Beijing (100700), China

Correspondence to: Prof. CHEN Ke-ji, Tel/Fax: 86-10-62880665,

E-mail: chen.keji@gmail.com

DOI: $10.1007 / \mathrm{s} 11655-009-0152-6$ 